Proc. Indian Acad. Sci. (Earth Planet. Sci.), Vol. 105, No. 2, June 1996, pp. L191-L195.

(C) Printed in India.

\title{
Modelling of soil temperature profiles using data from the pilot experiment at Anand
}

\author{
B JOSEPH and P GOSWAMI \\ CSIR Centre for Mathematical Modelling and Computer Simulation, Bangalore 560037, \\ India \\ MS received 5 April 1996
}

\begin{abstract}
We present some preliminary results from a multi-layer soil temperature finitedifference model using the data set obtained during the pilot experiment on land surface processes at Anand. We hope that the present results will prove useful during the final field experiments scheduled for the coming monsoon season.
\end{abstract}

Keywords. Soil temperature; land surface processes; land surface experiment; soil process modelling.

\section{Introduction}

Over the past few years there has been a growing realization that the land surface processes play important roles in atmospheric circulation at various scales. The exchanges of momentum, heat and moisture between the atmosphere and the underlying surface strongly influence the dynamics and thermodynamics of the atmospheric system. Thus an accurate representation of these processes is essential for improving numerical weather prediction (NWP). Many of the land surface parameters are also crucial for designing agricultural strategies. This is particularly true for India whose economy depends strongly on the country's agricultural prospects. As NWP came of age and operational agrometeorological forecasts became a possibility, the atmospheric scientists became increasingly aware of an urgent need for modelling and incorporating this important aspect in NWP models. However, lack of an appropriate data set based on observations for the Indian region was a major bottleneck in this direction.

To fill this gap between modelling and observation, the Department of Science and Technology (DST) has planned to carry out a major field experiment of land surface processes. The main site of the experiment selected is Anand, Gujarat. Although the main experiment is scheduled for 1996, a pilot experiment has already been completed for the period April to July 1995 at the Gujarat Agricultural University campus by the scientists of the Indian Institute of Tropical Meteorology, Pune, and their collaborators. Since these experiments provided measurements of several variables at frequent intervals, this data set provided an excellent opportunity to validate a dynamical model for the land surface processes. Such an effort has been initiated at the CSIR Centre for Mathematical Modelling and Computer Simulation, Bangalore. In the first phase of this effort a one-dimensional model for the soil variables has been developed. The purpose of this note is to communicate quickly to the scientific community certain results from the modelling study which might prove useful for the final experiment to be 
carried out in the coming months of the current year. In particular, we emphasize on the modelling of soil temperature profiles. Apart from its significance in NWP, the temperature of a soil greatly affects the physical, chemical and biological processes occurring in that soil which are crucial for the plant life (cf. Brady 1990).

\section{The model}

We use a one-dimensional multi-layer soil model with temperature as the basic dynamic variable. Moisture is not included as a model variable in the present version because the pilot experiment data do not contain data on soil moisture. From the requirement of conservation of heat, the time evolution of soil temperature $T$ is given by

$$
\rho_{s} c_{s} \frac{\partial T}{\partial t}=-\frac{\partial F}{\partial z}
$$

where $F$ denotes the soil heat flux, $\rho_{s}$ is the soil density and $c_{s}$ is soil specific heat. The subsurface heat flux $F$ is given by the Fourier law of heat conduction

$$
F=-\lambda \frac{\partial T}{\partial z}
$$

where $\lambda$ is the thermal conductivity of the soil $\left(\lambda=\rho_{s} c_{s} \kappa_{s}\right.$, where $\kappa_{s}$ is the thermal diffusivity). In general, $\lambda$ is a function of depth and other variables (e.g., soil type and moisture) and varies by an order of magnitude between dry soil and rock ( 0.25 to $2.9 \mathrm{~W} \mathrm{~m}^{-1} \mathrm{~K}^{-1}$ ) (cf. Garratt 1992). In our model, we had assumed a prescribed profile in depth for $\lambda$.

Equations (1) and (2) are solved numerically using an explicit time integration scheme starting from a prescribed initial temperature profile. The initial profile was derived from the observed profile at the time of beginning the model integration using linear interpolation between observation levels. The boundary condition (BC) at the bottom of the soil layer is zero heat flux and that at the top (surface) is either fixed surface temperature or fixed surface heat flux, both of which are available in the Anand data set. The model uses variable resolution in the vertical with highest resolution near the surface. The depth of the soil layer in the model is $1.5 \mathrm{~m}$. The top $10 \mathrm{~cm}$ of the soil layer (we call it layer-1) is divided into 20 sublayers, the next $20 \mathrm{~cm}$ (layer-2) is divided into 10 sublayers, the next $20 \mathrm{~cm}$ (layer-3) into 8 sublayers and the last $100 \mathrm{~cm}$ (layer-4) also into 8 sublayers. It should be mentioned that the deepest level in the pilot experiment data is at $1 \mathrm{~m}$. In the case when flux is used as the upper BC, all the flux points are at the interfaces of the sublayers and all the temperature points are at the midlevels (or half-levels) of each sublayer and vice versa if temperature is used as the upper BC. The profile of $\lambda$ has been chosen such that the heat conductivity is assumed to decrease with depth. This is a reasonable assumption if the soil moisture also decreases with depth. It is well-known that the conductivity of heat from soil to water is about 150 times more than that from soil to air (cf. Brady 1990). Once the pores in the soil get filled with water, the air content decreases and the heat transfer resistance is lowered very much and so values of $\lambda$ can be expected to be larger in wet soil. The values of $\lambda$ used in this study are $2 \cdot 2,2 \cdot 2,1.8$ and $1.6\left(\mathrm{Wm}^{-1} \mathrm{~K}^{-1}\right)$ in layers $1,2,3$ and 4, respectively. 

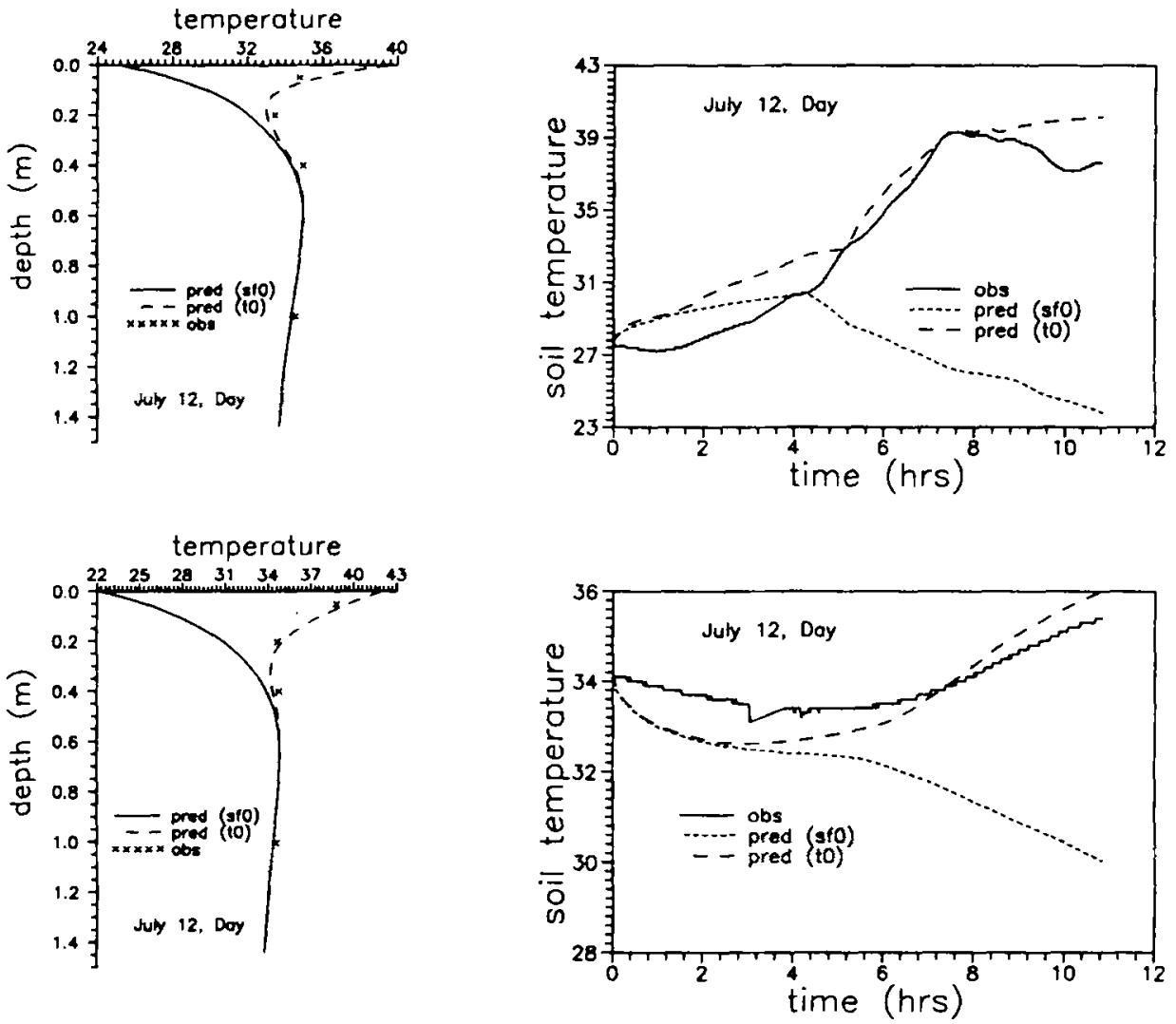

Figure 1. Model simulation for the day of 12 th July. The time integration starts at $0531 \mathrm{hr}$ IST. The left panels show the vertical profiles at the 6th hour (upper) and 9 th hour (lower). The right panels show the temporal behaviour of the model at depths 5 and $20 \mathrm{~cm}$. In all the panels the temperature is in degree Celsius.

The model is integrated by updating the upper boundary condition every $10 \mathrm{~min}$ using the observed data. The model time-step has been chosen to be $5 \mathrm{sec}$, consistent with the CFL criteria.

\section{Results}

Figures 1 and 2 show two sample simulations with the model, one for the day of 12th July and the other for the night of 24th July. In each of these figures, the left panels show the soil temperature profiles after $6 \mathrm{hr}$ (upper) and $9 \mathrm{hr}$ (lower) of model integration. The solid lines in these panels represent simulated values with the top $B C$ given in terms of surface heat flux while the dashed line represents simulation with the top BC determined by surface temperature. The observed values at the depths at which they are available are represented by cross symbols. The right panels in the figures show the predicted and observed temperatures as a function of time, at depths $5 \mathrm{~cm}$ (upper) and $20 \mathrm{~cm}$ (lower). In these panels, the solid line represents 

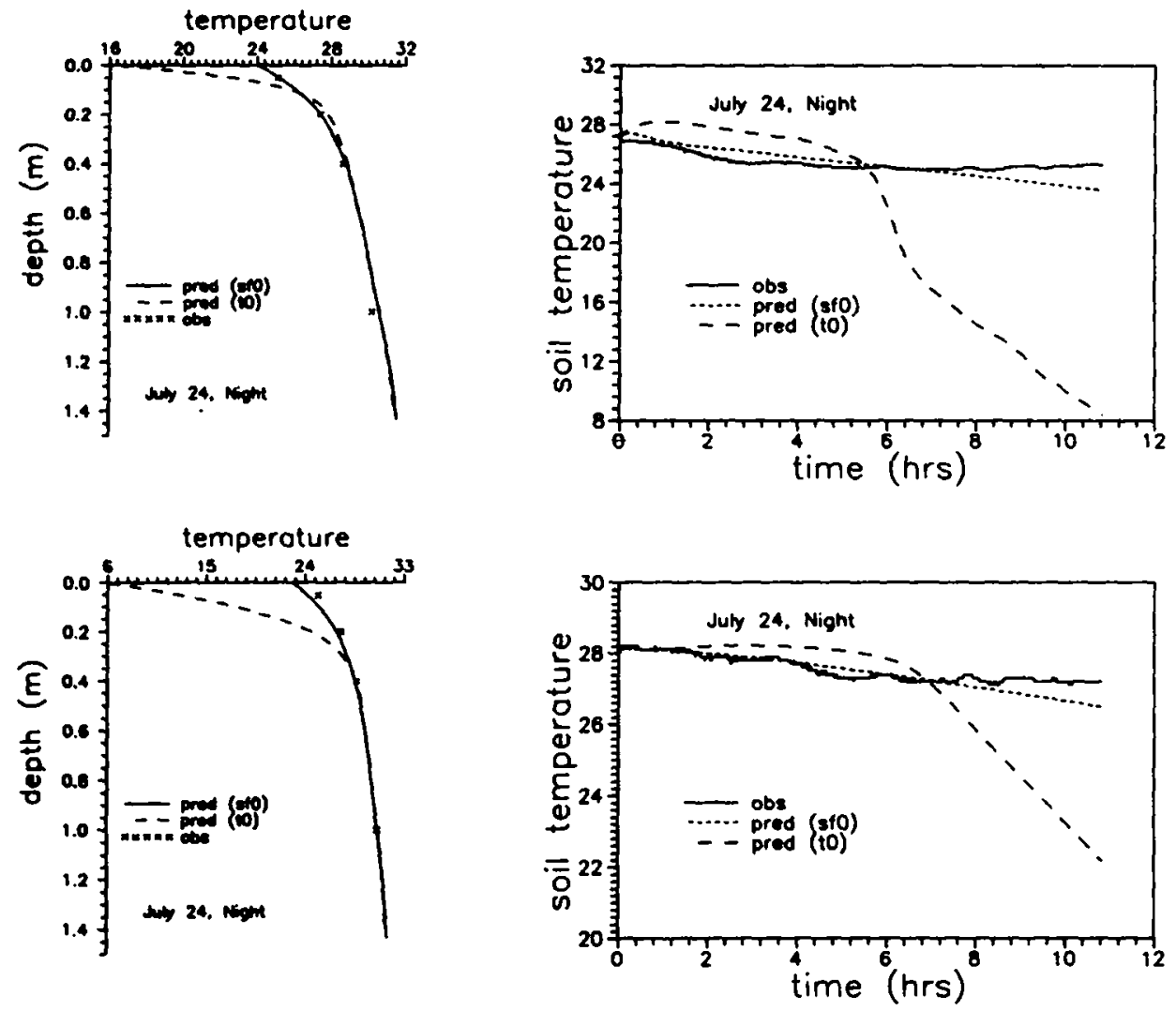

Figre 2. Same as figure 1, but for the night of 24 th July. The time integration starts at $1731 \mathrm{hr}$ IST.

the observed temperatures, while the small-dashed and big-dashed lines represent the simulated values with soil fiux as upper $\mathrm{BC}$ and temperature as upper $\mathrm{BC}$, respectively.

As can be seen from figure 1, the prescription of the top BC in terms of temperature seems to provide a much better day-time simulation on 12th July. However, the night-time simulation on 24th July (figure 2) shows that it is the prescription of flux as the upper BC which provides a better simulation. This is exactly the point which we wish to emphasize in this note. It should be mentioned that we have used a first-order scheme for calculation of the fluxes, and the result is unlikely to be due to any numerical effect. Further scrutiny of the observations revealed that the period of simulation for which flux boundary conditions provided better simulations were preceded by a substantial amount of rainfall.

Two other simulations (day and night of June 8th, 1995) with the same model (not shown) also agree qualitatively to our above finding that the flux BC gives better simulations during night and the temperature $B C$ gives better simulation during the day. However, the performance of the model with flux as BC was far superior for the night-time simulation of July $24 \mathrm{th}$, which was preceded by rain. Thus, from a modelling view-point, observations of both flux and temperature are important. 
It also appears likely that the inclusion of some physical processes and a corresponding observational parameter, such as the cloud cover, will be necessary to account for the above mentioned effect. Finally, it must be emphasized that the present set of simulations needs to be subjected to a more rigorous statistical evaluation. However, we found the effect persistent and interesting enough to bring it to the notice of the scientific community.

\section{Acknowledgements}

The authors would like to thank Dr. K G Vernekar, IITM, Pune, for providing the Anand pilot experiment data set.

\section{References}

Brady N C 1950 The nature and properties of soil (Singapore: Macmillan Pub. Co.)

Garratt J R 1992 The atmospheric boundary layer (Cambridge: Cambridge University Press) 